\title{
Transversalización de la Promoción de la Salud en el Currículo de Estudios de la Universidad Nacional José Faustino Sánchez Carrión - 2013
}

\author{
Mainstreaming health promotion in the curriculum of studies at the National \\ University José Faustino Sanchez Carrion - 2013 \\ Elsa Carmen Oscuvilca Tapia ${ }^{1, a}$, Walter Jesús Sosa Hijar ${ }^{1, b}$, \\ William Rogelio Peña Ayudante ${ }^{1, c}$, Jacinto Jesús Palacios Solano ${ }^{1, d}$
}

\section{RESUMEN}

Objetivo: Determinar el efecto del programa formativo sobre transversalización de la promoción de salud en el curriculo de estudios de la Universidad Nacional José Faustino Sánchez Carrión. Materiales y Métodos: Estudio Cuasi-Experimental, longitudinal y prospectivo realizado en una muestra de 61 docentes, en el que se desarrolló un programa formativo sobre promoción de salud; para el analisis de los datos se empleó la prueba t de Student con el programa SPSS versión 17.0. Resultados: El 60\% de las carreras profesionales, cosideran contenidos sobre promoción de salud en el plan de estudios. Los docentes del grupo control en el pre y post test tienen el conocimiento regular y bajo el $80 \%$; del grupo experimental en el pre test $35 \%$ tienen conocimiento regular y bajo; en el post test el $48 \%$ presentan el conocimiento alto. En conclusión el programa formativo sobre transversalización de la promoción de salud en el currículo de estudi

Palabras Claves: Riesgos. Factores. Deserción Estudiantil.

\begin{abstract}
Objective: To determine the index that generates the risk of desertion of students of the Faculty of Science, National University José Faustino Sanchez Carrion and the main cause that generates the risk of desertion. Methods: We evaluated the Individual Factors; Socioeconomic: institutional, academic, Economic, Social, Health and Academic, related to performance and professional careers satisfaction, based on the methodology proposed in the article: Construction of student drop index University using the theory Rationale limited. This has been adapted to the reality of our University. Results: We have obtained that the predominant factor that generates risk of desertion of the Career in students of Applied Mathematics and Statistics and Informatics is the Academic factor related to satisfaction with professional career, for students of Biology with mention in Biotechnology, is the Institutional Aspect related to infrastructure and adaptation environment for students. Conclusions: The desertion index is influenced by several factors and this depends on the problems faced by each student in his professional career.
\end{abstract}

Keywords: Risks. Factors. Student desertion.

\footnotetext{
${ }^{1}$ Facultad de Medicina Humana, Universidad Nacional José Faustino Sánchez Carrión, Huacho, Perú. ${ }^{a}$ Docente Principal. Doctor en Salud Pública, ${ }^{b}$ Docente Asociado. Lic. en Bromatología y Nutrición, ' Docente Asociado. Maestro en Salud Reproductiva, ${ }^{\mathrm{d}}$ Docente Principal. Magister en Fisiología.
} 


\section{INTRODUCCIÓN}

Desde la $6^{\circ}$ Conferencia Mundial de Promoción de la Salud, realizada del 7 al 11 de agosto del 2005, en Bangkok, Tailandia, la transversalización de la promoción de la salud es un componente primordial de la agenda de desarrollo mundial, que las comunidades y sociedad civil lo asuman como un objetivo fundamental y constituya como requisito de buenas practicas empresariales ${ }^{(1,2)}$, siendo las Universidades espacios de formación integral de los profesionales, desde el punto de vista humanístico, científico y tecnológico, a través de una gestión basada en los principios del desarrollo humano sostenible; porque son instituciones sanas socialmente ${ }^{(3)}$ porque incorporan las áreas de gestión de la promoción de la salud en los fines de la universidad.

Tal es asi que en las mallas curriculares de formación academica se debe transversalizar cursos y/o módulos formativos con contenidos de promoción de la salud: conservación del ambiente, sexualidad, habilidades para la vida, prevención del consumo drogas, tabaquismo y alcohol, hábitos saludables en alimentación y nutrición.

Ello permite al docente actuar con pertinencia y compartir una labor interdisciplinaria, permeabilizando la dinámica sociocultural y académica.

Las experiencias de programas de salud en las universidades, a nivel nacional son numerosas y con modalidades diversas que desarrollan como parte de las funciones de Bienestar Universitario con actividades asistenciables y preventivo promocionales con distintos niveles de aplicación y cobertura social; donde las demandas internas de salud universitaria son atendidas a través de los servicios de medicina general, salud dental, salud mental, salud de la gestante. primeros auxilios, entre otros; como una pertinente expresión de su rol social basados en los principios del desarrollo humano explícitos en los estandares 87,88 y 89 factor 7 del Modelo de Calidad para la Acreditación de Carreras Profesionales Universitarias establecidas por Ley $\mathrm{N}^{\circ}$ 28740. La presente investigación es relevante en lo social, aporte el valor teórico debido a que la transversalización permite el abordaje transdiciplinario ante la necesidad de desarrollar un marco establecido por la Organización Mundial de la Salud, que se encuentra en implementación en el contexto universitario a nivel nacional, latinoamericano y mundial, puesto que la solución comprende componentes tanto empíricos como teóricos, constituye sin lugar a dudas una contribución al conocimiento multidisciplinar y por las implicancias prácticas; por ser una iniciativa que enfatiza el compromiso de acreditar profesionales que se incorporen al mercado laboral con las máximas garantías de una buena formación, complementando con la responsabilidad social de ofertar a la ciudadanía las posibilidades de un aprendizaje a lo largo de toda la vida.
Se sustenta en las bases teóricas del aprendizaje social de Albert Bandura y el Modelo Promotor de Salud de Nola Pender.

En base a lo que viene aconteciendo en similares instituciones universitarias y considerando los supuestos teóricos y prácticos de la UNESCO ${ }^{(6)}$ y de la OMS, se realiza la investigación, cuyo objetivo fue determinar el efecto de la transversalización de la promoción de salud en el currículo de estudios y en el conocimiento de los docentes de la Universidad Nacional José Faustino Sánchez Carrión.

\section{MATERIALES Y MÉTODOS}

Tipo de estudio Cuasi-Experimental (diseño con grupo experimental y control), corte longitudinal y prospectivo. El área de estudio, las Escuelas Académicos Profesionales de la Universidad Nacional José Faustino Sánchez Carrión. La población constituida por 600 docentes y el tamaño de muestra 61 docentes determinados mediante la formula de Cochran $^{(7)}$ basada en una variable auxiliar con una proporción.

Donde:

$$
\mathrm{n}=\frac{\frac{z^{2} p q}{d^{2}}}{1+\frac{1}{N}\left(\frac{Z^{2} p q}{d^{2}}-1\right)}
$$

z: Valor de lá unuvisu un iи uisu inuviun normal de dos colas, que nos indica la probabilidad en la cual se halla la proporción $p$ buscada. 1.64 (nivel de confianza del 90\%)... [2]

d: Error máximo permisible. Este es igual: $d=e p=$ $0.08 \ldots[3]$

\section{Donde:}

e: Error relativo $10 \%(e=0.10) \ldots$

p: Proporción observable $(80 \%) \ldots$ [4]

q: 1-p. Esto significa la proporción no observable (20\%)...[5]

$\mathbf{N}$ : Tamaño de la población objetivo (600 docentes)...[6]

n: Tamaño de la muestra

Con los valores numéricos de [2],[3],[4],[5],[6], y utilizando la ecuación de Cochran [1] se procedió a construir una tabla de doble entrada según confiabilidades muestrales (filas) y Errores Relativos (columnas) para conocer los diferentes tamaños de muestra.

Puesto que los cálculos se basaron en un análisis exhaustivo de confiabilidad y validez, estos aseguraron escoger también niveles altos de confiabilidad muestral. De aquí que se determino el tamaño de muestra de 61 docentes que corresponde a un nivel de confianza muestral de $90 \%$ y un error 


$$
n=\frac{\left(\frac{(1.64)^{2} *(0.80 * 0.20)}{(0.08)^{2}}\right)}{1+\frac{1}{600}\left[\frac{(1.64)^{2} *(0.80 * 0.20)}{(0.08)^{2}}-1\right]} \quad n=\frac{67.2344}{1+\frac{66.2344}{600}} \quad n=61 \text { docentes }
$$

Tipo de muestreo, probabilistico, aleatorio al azar simple. El criterio de inclusión docentes que accedieron participar en la investigación y el de exclusión los docentes que no aceptaron participar en la investigación. Las técnicas e instrumentos de recolecta de datos, el instrumento sobre contenidos de promoción de salud y la ficha de observación para el análisis documental de los planes de estudio de las carreras profesionales, el programa formativo de transversalización de currículo.

Para la recolección de datos se realizaron los tramites administrativos en las direcciones de Escuela, determinando la fecha de inicio para la recolección de datos y el tiempo de duración de la recolección.

Técnicas para el procesamiento de la información: Se elaboró la base de datos en el software Excel y en el software estadístico SPSS versión 17.0 para Windows XP, incorporando los resultados en tablas y gráficos.

Los puntos de corte de la variable conocimiento en el grupo control y experimental fueron determinados según la escala de estaninos, siendo que:

$$
\begin{aligned}
& \bar{x} \pm 0.75 * D S \\
& \text { Dónde: } \\
& \bar{X}: \text { Media Aritmetica } \\
& \text { DS: Desviación Estándar }
\end{aligned}
$$

Los resultados fueron expresados en porcentajes. Se aplicó la prueba parametrica $\mathrm{t}$ de Student para establecer la diferencia entre los grupos.

Tabla 1. Conocimiento del grupo control, pre y post test.

\begin{tabular}{lcccc}
\hline \multirow{2}{*}{ Conocimiento } & \multicolumn{4}{c}{ Grupo Control } \\
& Pre test & Post test \\
\hline & $\mathrm{n}$ & $\%$ & $\mathrm{n}$ & $\%$ \\
Bajo & 12 & 40 & 12 & 40 \\
Regular & 12 & 40 & 12 & 40 \\
Alto & 6 & 20 & 6 & 20 \\
$\quad$ Total & 30 & 100 & 30 & 100 \\
\hline
\end{tabular}

Fuente: Docentes UNJFSC 2013

La prueba $t$ de Student para la diferencia de medias (muestras relacionadas) pre test y post test en el grupo control:

$\mathrm{H}_{\mathrm{o}}$ : No existen diferencias entre las medias del pre test y post test en el grupo control.

$\mathrm{H}_{1}$ : Existen diferencias entre las medias del pre test y post test en el grupo control.

Nivel de significancia: Error tipo I, $\alpha=0.05$ (5\%). Esto es, el error que se cometeria al rechazar la $\mathrm{H}_{\mathrm{o}}$, siendo esta verdadera.
Prueba Estadistica Paramétrica: Prueba t de student.

\begin{tabular}{|c|c|c|c|c|c|c|c|c|}
\hline & & Dif & erencia & e Media & & & & \\
\hline \multirow{2}{*}{$\begin{array}{c}\text { Pre - Post } \\
\text { test }\end{array}$} & \multirow{2}{*}{ Media } & \multirow{2}{*}{$\begin{array}{c}\text { Desviacións } \\
\text { td }\end{array}$} & \multirow{2}{*}{$\begin{array}{l}\text { Std. } \\
\text { Error } \\
\text { Media }\end{array}$} & \multicolumn{2}{|c|}{$\begin{array}{c}95 \% \text { Intervalo } \\
\text { Confianza para la } \\
\text { Diferencia }\end{array}$} & \multirow[b]{2}{*}{$\mathrm{T}$} & \multirow{2}{*}{\multicolumn{2}{|c|}{ GL $\begin{array}{l}\text { Sig. (2 } \\
\text { tailed) }\end{array}$}} \\
\hline & & & & Inferior & Superior & & & \\
\hline & -0.033 & 0.183 & 0.033 & -0.102 & 0.035 & -1.000 & 29 & 0.326 \\
\hline
\end{tabular}

Estadística Muestras relacionadas

\begin{tabular}{lcccc}
\hline & Media & $\mathrm{N}$ & Desviación Std & Error Std. Media \\
\hline Pre test & 22.23 & 31 & 2.012 & 0.367 \\
Post test & 22.27 & 31 & 1.964 & 0.359 \\
\hline
\end{tabular}

Test Muestras relacionadas

Diferencia de Medias

Desviación Estadística:

La diferencia de medias observadas es de -0.033 , asi mismo se observa que el $E E=0,033$. El intervalo de confianza al $95 \%$ para la diferencia de medias nos da un limite inferior de -0.102 y un limite superior de 0.035 (es importante notar que este intervalo de confianza incluye al valor 0 ). Nuestro T estadístico calculado es de -1.000 con $G L=29$, al cual le está asociado un valor de probabilidad, $\mathrm{P}=0.326$. Puesto que este valor $\mathrm{P}$ es mayor que $\alpha(0.05)$, se concluye en aceptar la $\mathrm{H}_{0}$.

Es decir no existen diferencias significativas entre las medias del pre test y post test en el grupo control.

El conocimiento del grupo experimental sobre transversalización de la promoción de salud en el curriculo de estudios se muestra en la tabla 2.

Tabla 2. Conocimiento del grupo control, pre y post test.

\begin{tabular}{lcccc}
\hline \multirow{2}{*}{ Conocimiento } & \multicolumn{4}{c}{ Grupo Control } \\
& Pre test & Post test \\
\hline & $\mathrm{n}$ & $\%$ & $\mathrm{n}$ & $\%$ \\
Bajo & 11 & 35.4 & 6 & 19.3 \\
Regular & 10 & 32.3 & 10 & 32.3 \\
Alto & 10 & 32.3 & 15 & 48.4 \\
Total & 31 & 100 & 31 & 100 \\
\hline
\end{tabular}

Fuente: Docentes UNJFSC 2013

La prueba $t$ de student para la diferencia de medias (muestras relacionadas) pre test y post test en el grupo experimental:

$\mathrm{H}_{\mathrm{o}}$ : No existen diferencias entre las medias del pre tst y post test en el grupo experimental.

$\mathrm{H}_{1}$ : Existen diferencias entre las medias del pre test y post test en el grupo experimental.

Nivel de significancia: Error tipo I, $\alpha=0.05$ (5\%). Esto es, el error que se cometería al rechazar la $\mathrm{H}_{\mathrm{o}}$, siendo esta verdadera.

Prueba Estadística Parametrica: Prueba t de Student.

Estadística Muestras relacionadas

\begin{tabular}{lcccc}
\hline & Media & $\mathrm{N}$ & $\begin{array}{c}\text { Desviación } \\
\text { Std }\end{array}$ & $\begin{array}{c}\text { Error } \\
\text { Std. Media }\end{array}$ \\
\hline Pre test & 22.58 & 31 & 2.187 & 0.393 \\
Post test & 31.00 & 31 & 1.612 & 0.290 \\
\hline
\end{tabular}




\section{Test Muestras relacionadas}

Diferencia de Medias

\begin{tabular}{|c|c|c|c|c|c|c|c|c|}
\hline \multirow{2}{*}{$\begin{array}{c}\text { Pre - Post } \\
\text { test }\end{array}$} & \multirow[t]{2}{*}{ Media } & \multirow{2}{*}{$\begin{array}{l}\text { Desviacións } \\
\text { td }\end{array}$} & \multirow{2}{*}{$\begin{array}{l}\text { Std. } \\
\text { Error } \\
\text { Media } \\
\end{array}$} & \multicolumn{2}{|c|}{$\begin{array}{c}\text { 95\% Intervalo } \\
\text { Confianza para la } \\
\text { Diferencia }\end{array}$} & \multirow[b]{2}{*}{$\mathrm{T}$} & \multirow[b]{2}{*}{$\mathrm{GL}$} & \multirow{2}{*}{$\begin{array}{l}\text { Sig. (2- } \\
\text { tailed) }\end{array}$} \\
\hline & & & & Inferior & Superior & & & \\
\hline & -8419 & 2.718 & 0.488 & -9.416 & -7.423 & -17.250 & 30 & 0.000 \\
\hline
\end{tabular}

Decisión Estadística: La diferencia de medias observa que el $E E=0,488$. El intervalo de confianza al $95 \%$ para la diferencia de medias nos da un limite interior de -9.416 y un limite superior de -7.423 (es importante notar que este intervalo de confianza no incluye al valor 0). Nuestro T estadistico calculado es de -17.250 con $\mathrm{GL}=30$, al cual le está asociado un valor de probabilidad, $\mathrm{P}=0.000$.

Puesto que este valor $P$ es menor que $\alpha(0.05)$, se concluye en rechazar la $\mathrm{H}_{0}$.

\section{Es decir existen diferencias significativas entre las medias del pre test y post test en grupo experimental.}

\section{DISCUSIÓN}

Para alguno autores transversalizar la promoción de salud en el currículo de estudios significa institucionalización de la promoción de salud, debido a que las instituciones son las reglas de juego en una sociedad, son las limitaciones ideadas por el hombre que dan forma a la interacción humana. Las instituciones estructuran incentivos en el intercambio humano: político social y económico. El cambio institucional conforma el modo en que las sociedades evolucionan a lo largo del tiempo, clave para entender el cambio histórico. Las instituciones reducen la incertidumbre dan estructura a la vida diaria; constituyen una guía para la interacción humana para realizar actividades ${ }^{(8)}$.

La transversalidad surge como una propuesta por la comisión sobre la condición jurídica y social de la mujer de las Naciones Unidas. Posteriormente fue asumido explícitamente como estrategia global dentro del marco del III Programa de acción comunitaria para la igualdad de género. La transversalizaciónes un proceso que recorre el currículo con tenidos culturalmente relevantes y necesarios para el cuidado de la salud (conservación del ambiente, ejerccico fisico, sexualidad, habilidades para la prevención del consumo de drogas, tabaquismo, alcohol y hábitos saludables en alimentación y nutrición) deben estar presentes en todo el proceso de formación profesional. Son temas que no necesariamente tienen que conformar una asigantura en particular ni recibir un tratamiento especial dentro del currículo, sino que deben abordarse en todas las áreas que lo integran y en toda situción concreta de aprendizaje sustentados en el marco conceptual del Modelo Promotor de Salud de Penser y de la teoría del aprendizaje social de Albert Bandura.

En la presente investigación, los docentes del grupo control, el $83 \%$ correspondieron al sexo femenino, el $17 \%$ de sexo masculino. La edad promedio fue de 34 años, la información sobre la procedencia el $40 \%$ de Lima, y $63.3 \%$ viven con su familia.

Los Docentes del grupo experimental, el $71 \%$ correspondieron al sexo femenino el $29 \%$ de sexo masculino. La edad promedio fue de 49.84 años, el $42 \%$ proceden de Lima y el $54.8 \%$ viven con su familia. En el pre test el $32.3 \%$ tiene el conocimiento regular y alto, el post test el $48 \%$ tiene conocimiento alto, el mismo que implica conceptualizar el significado de transversalización del currículo, establecer la necesidad de desarrollar el perfil de un profesional promotor de salud, priorizar la secuencia y mantener la coherencia a lo largo de los distintos semestres académicos (lógica vertical) y al mismo tiempo coherente dentro de un mismo periodo (lógica horizontal).

Se determinó que, 20 carreras $(60 \%)$ de la Universidad Nacional José Faustino Sánchez Carrión consideran en su plan de estudios asignaturas o contenidos de promoción salud, 48 asignaturas son obligatorias y 06 asignaturas electivas, ubicadas en los primeros años de formación profesional, no tienen la condición de pre requisitos y ninguna de ellas considera a la promoción de salud, como eje transversal, en virtud a ello grupo experimental sugirió la elaboración de sumillas con ejes temáticos de promoción de salud, a cargo de las especialidades, por citar la carrera de Ingeniería civil, debe asumir la conservación de la infraestructura física; Educación física y deportes, el ejercicio físico, la salud y la calidad de vida; Medicina Humana y Enfermería, la prevención del consumo drogas, tabaquismo y alcohol, promoción de conductas saludables y generación de espacios saludables, la sexualidad (identidades y los papeles de género, el erotismo, el placer, la intimidad, la reproducción y la orientación sexual; Bromatología y Nutrición, los hábitos saludables en alimentación y nutrición.

Lo descrito no coincide con la transversalización de la promoción de salud realizada en la Universidad Peruana Unión el fomento de la prevención de enfermedades y desarrollo de asignaturas de cultura física y educación para la salud en las mallas curriculares en todas las carreras profesionales como parte de la formación general, responde al principio de la salud como lineamiento universitario. La Universidad Metropolitana de Honduras desde el 2009 , cuenta con la guía para transversalizar el eje ambiental en el nivel de educación superior. La Universidad del Norte Santo Tomás de Aquino de Tucumán, Argentina, incorpora las acciones de promoción de la salud a partir del 2008, para propiciar el desarrollo humano y mejorar la calidad de vida de sus integrantes a través de los promotores de conductas saludables introduciendo los contenidos de salud en los currículos y procesos educativos.

Carmen Gallardo Pino concluye que, la transversalización de promoción de la salud tiene efectos positivos debido a que el Vicerrectorado de 
Política Social, Calidad Ambiental y Universidad Saludable de la Universidad Rey Juan Carlos (Madrid, España) es política institucional.

María Eugenia Uribe destaca que, los espacios saludables al interior de las universidades (propicios para el consumo de alimentos sanos y la práctica de la actividad física, espacios $100 \%$ libres de humo, disminución del consumo de sustancias psicoactivas y de alcohol) debe operar a través de asignaturas de libre elección y/o a través de los líderes o representantes estudiantiles.

Mercedes MarzánDelís el programa de Universidades por la salud, consiste en mejorar la transversalización de la promoción de salud en las carreras, tanto de las ciencias de la salud, incluidas las médicas y no médicas. Uno de los tantos proyectos comunitarios pioneros fue el de "Sueño sin mosquitos", que perseguia involucrar a las comunidades en la lucha contra el dengue, desde el enfoque multidisciplinario.

En conclusión el programa formativo sobre transversalización de la promoción de salud en el curriculo de estudios, tiene efecto significativo en el grupo experimental, sugiriendo el cambio de paradigmas en salud para institucionalizar la promoción de salud.

Conflicto de interés: Los autores declaran no tener conflictos de interés de ninguna naturaleza.

\section{AGRADECIMIENTO}

Los investigadores agradecen por su colaboración al Mstro. Melchor Escudero Escudero por su contribución en la corrección de estilo y aplicación del instrumento de recolecta de datos. A la Lic. Norvina Marlena Angulo y Dr. Armando Vásquez Aristizabal por colaboración en aplicación del instrumento de recolecta de datos. 


\section{REFERENCIA BIBLIOGRÁFICA}

Organización Mundial de la Salud. Organización Panamericana de Salud. Determinantes de la Salud. Ginebra . [citado 26 Setiembre 2012].

Ministerio de Salud Dirección General de Promoción de la Salud Promoviendo Universidades Saludables. Lima Perú, 2010.

Guerra A. Modelo en crisis y ofensiva estudiantil.2011. Chile [citado15 noviembre 2013].

Centro Nacional de Actualización Docente Generación XXIX Secuencias didácticas. SUB SECRETARÍA DE EDUCACIÓN MEDIA SUPERIOR. México. [citado 29 Setiembre 2013].

Bandura Albert. Teorias de la personalidad. traducción, Rafael Gautier, 2002 [citado 26 Setiembre 2013]

UNESCO. Quinta Conferencia Internacional de Educación de las Personas Adultas (CONFINTEA V), Hamburgo, 1997.

Cochran G. Tecnicas de Muestreo. CECSA.

Dussan Ana C. Instituciones cambio institucional y desempeño económico. Douglas North. [citado 2 Setiembre 2013].

Naciones Unidas para la igualdad de genero y el empoderamiento de las mujeres.

Redon S. significados de la transversalidad en el curriculum: Un estudio de caso Escuela de Pedagogia, Facultad de Filosofia y Educación Pontificia Universidad Católica, Chile. Revista
Iberoamericana de Educación (ISSN: 1681 - 5653) $n^{\circ} 43 / 2$ - 10 de junio de 2007. Organización de Estados Iberoamericanos para la Educación, la Ciencia y la Cultura.

Ministerio de Salud. Dirección General de Promoción de la Salud. Dirección de Educación para la Salud Documento técnico: Promoviendo universidades saludables. Lima 2010.

International ResourcesGroup (IRG) y AGA \& Asociados-Consultores en Comunicación, "Guia para transversalizar el eje ambiental en las carreras del nivel de Educación Superior de Honduras" 2009.

Una nueva Mirada al Movimiento de Universidades Promotoras de la Salud en las Américas, Documento de Trabajo del IV Congreso Internacional de UPS, Universidad Pública de Navarra 2009.

Gallardo C., Martínez A. Promoción de la salud en la universidad: la Universidad Rey Juan Carlos, una universidad saludable. Vicerrectora de Politica Social, Calidad Ambiental y Universidad Saludable. Madrid. España. 2008.

Uribe E. Transversalización de la educación. Universidad Libre de Cali. Red Colombianan de Instituciones de Educación Superior Promotoras de Salud. [citado 2 mayo 2013]

Marzán M.; Bonal F. Fortaleciendo la Universidad por la salud. Centro Provincial de Promoción y Educación para la salud, Santiago de Cuba, MEDISAN vol. 16 nro 2 . 2012. 